\title{
Rosmarie Barwinski (2020): Steuerungsprozesse in der Psychodynamischen Traumatherapie. Stuttgart: Klett-Cotta
}

\author{
Lutz Wittmann (Berlin)
}

Mit welch beneidenswerter Unbeschwertheit konnten sich doch die Pioniere* der Psychoanalyse ans Schreiben ihrer Bücher machen. Doch seither gilt für alle ihre Nachfahren, was Kurt Tucholsky 1931 in der Weltbühne konstatierte: Es gibt keinen Neuschnee. Die große Herausforderung besteht also darin, zunächst ganze Berge verwandter Literatur zu überblicken, das Relevante zu erkennen, und die vielen komplizierten Theorien, deren Darstellung sich im Original oft mehr durch Ausführlichkeit denn durch Verständlichkeit auszeichnet, in aller Kürze wiederzugeben. Quasi zur Einleitung, denn dann soll es ja weitergehen. Was Barwinski hier gelingt, ist nicht weniger als ein Lehrstück. Mit unprätentiöser Schlichtheit bietet Teil 1 dem Leser eine an Klarheit kaum zu übertreffende Einführung in die Gedächtnispsychologie, die Ausführungen von Symbolisierungstheoretikern wie Charles Peirces, Anna Aragno oder Heinrich Deserno, die kognitive Entwicklung nach Piaget, oder das britische Mentalisierungskonzept. Die Anschaulichkeit bleibt dabei auch dank des systematischen Einsatzes kleiner Fallvignetten erhalten. So wird die Piste für die zu unternehmende Abfahrt mit einer tragenden Decke Altschnees vorbereitet. Ausgehend von Aragnos Ebenen der symbolischen Organisation entwirft Barwinski sechs Symbolisierungsstufen, welche der Integrationsprozess traumatischer Erfahrungen auf der Buckelpiste vom somatoformen Symptom zum vollständigen Narrativ der eigenen Traumageschichte durchlaufen muss. Sie arbeitet also die denkwürdige Hypothese aus, dass «die Repräsentation traumatischer Erlebnisse ähnliche Stufen durchläuft, wie wir sie bei der Repräsentanzenbildung in der kindlichen Entwicklung finden» (S. 255). In Anlehnung an Haeckels Rekapitulationstheorie könnte diese Beschreibung des Heilungsverlaufs lauten: Die posttraumatische Salutogenese rekapituliert die kognitive Ontogenese. Das Stufenmodell ist im weiteren Darstellungsverlauf der strukturierende Dreh- und Angelpunkt, es dient als Koordinatensystem, dem alle relevanten Dimensionen zugeordnet werden können. Diese Dimensionen

* Aus Gründen der besseren Lesbarkeit wird im Text verallgemeinernd das generische Maskulinum verwendet 
umfassen charakteristische Abwehrformen, Mentalisierungsmodi, Stufen des IchBewusstseins, erkennbare Muster im Ausdruck von Phantasien und Konflikten, oder typische Übertragungs-Gegenübertragungs-Dynamiken.

Im zweiten Teil folgt Barwinski dem Berner Philosophen Thomas Kesselring in dessen Verknüpfung von Piagets kognitivem Entwicklungsmodell mit Hegels Dialektikverständnis und seiner Hypothese, «dass auf allen Entwicklungsstufen Antinomien Entwicklungsschübe auslösen oder die Entwicklung blockieren können» (S. 122). Sie beschreibt drei Antinomien zwischen spezifischen Symbolisierungsstufen, nämlich die Vermischung der sicheren Gegenwart mit der traumatischen Vergangenheit, den Widerspruch zwischen Traumaerinnerung und gespaltener Objektrepräsentanz des «guten» Täters (S. 131), und Konflikte zwischen Wünschen und Über-Ich-Regeln. Ausgehend von Fischers allgemeinem dialektischen Veränderungsmodell der Psychotherapie stellt Barwinski nun ein 5-Punkte-Programm vor, wie der Umgang mit diesen Antinomien unterstützt werden kann, sodass sie zum Motor für Eigenaktivität und Veränderung werden. Dieses Programm umfasst im Einzelnen (S. $138 \mathrm{ff.}$ ):

1. Problematisches Verhalten ausfindig machen (A).

2. Bestimmung eines Gegenpols zum problematischen Verhalten, der auch zu Schwierigkeiten führen kann (Z).

3. Suche nach positiven Komponenten, die in den beiden problematischen Beziehungsmustern enthalten sind (A' und Z').

4. Verknüpfung der beiden positiven Komponenten.

5. Suche nach einer Haltung, wie die Verknüpfung der beiden positiven umgesetzt werden könnte.

Indem Barwinski eingangs Piagets Modell der kognitiven Entwicklung mit der Mentalisierungstheorie kontrastierte, kann sie betonen: «Die für das Mentalisieren notwendigen Entwicklungsschritte spielen sich nicht primär entlang einer kognitiven Reifungslogik ab, sondern immer im Kontext von zwischenmenschlichen Beziehungen» (S. 256). Folgerichtig stellt sie «einem technikorientierten Vorgehen eine andere, beziehungsorientierte Sichtweise entgegen [...]» (S. 256). Dabei ist die Interventionsebene immer der Symbolisierungsstufe anzupassen.

Im dritten Teil fasst Barwinski neun der gängigsten Traumatherapieansätze in ihren jeweiligen Voraussetzungen, Zielsetzungen und Techniken zusammen. Das entwickelte Koordinatensystem erlaubt ihr, zu erkennen, welcher Ansatz bzw. welche seiner Komponenten für die Bearbeitung der unterschiedlichen Symbolisierungsstufen der traumatischen Erfahrung geeignet ist. Hier wird deutlich, wieviel umfassender die psychodynamischen Verfahren in ihrem Anspruch im 
Vergleich zu den typischerweise deutlich kürzeren, direktiveren, und manualisierten kognitiv-verhaltenstherapeutischen und anderen Ansätzen sind. Dennoch idealisiert sie die psychodynamischen Verfahren nicht, sondern hinterfragt die Bereitwilligkeit der beschriebenen Ansätze, reflexhaft auf Techniken aus Verhaltenstherapie oder EMDR zurückzugreifen.

Darf der Autor einer Rezension Wünsche für die zweite Auflage des zu besprechenden Buches vortragen? Und dann gleich vier Stück? Der drängendste Wunsch wäre ein detaillierterer Fallbericht in der Darstellung des 5-Punkte-Programms, sodass nicht nur die Entwicklung der eingeführten Themen beobachtbar, sondern auch die konkrete therapeutische Arbeit nachvollziehbarer wird. Der zweite Wunsch wäre hier und da eine zusätzliche Klärung der Evidenzbasis, auf welche sich die Autorin bezieht: wenn etwa bei komplex traumatisierten Patienten in manchen Therapieformen Retraumatisierungen befürchtet werden, nährt sich diese Sorge aus eigener Erfahrung, aus Patientenschilderungen, oder aus systematischen Studienergebnissen? Der dritte Wunsch wäre die Klärung, ob neben den drei veranschaulichten Antinomien auch weitere Widerspruchskonstellationen vorkommen. Vorstellbar wäre dies immerhin, denn: «jede Stufe entwickelt sich aus der vorhergehenden heraus, aber sie können nebeneinander aktiv sein» (S. 255). Und schliesslich wäre auch eine Einordnung zumindest zweier weiterer Therapiemethoden in das entwickelte Schema spannend. Mit diesen ist der international vielleicht bekannteste psychodynamische Ansatz, Mardi Horowitz' integrative psychodynamisch-kognitive Psychotherapie, gemeint, sowie das von Maryène Cloitre für die Behandlung komplex traumatisierter Patienten entwickelte, kognitiv-verhaltenstherapeutische Skills Training in Affective and Interpersonal Regulation/Narrative Therapy (STAIR/NT).

Zusammenfassend lässt sich sagen, dass der Wert von Barwinskis Beitrag gerade darin liegen mag, dass seine Autorin darauf verzichtet, das Rad neu zu erfinden und sich am Wettbewerb der Begriffsneuschöpfungen, der die Verständigung erschwert, ohne die Praxis zu befördern, zu beteiligen. In der Entwicklung des Eigenen aus dem Vorbestehenden heraus, in der Art und Weise, wie die vielen Vorarbeiten hochsystematisch in Bezug gesetzt werden, in diesem ausdifferenzierten Theoriesystem, welches nicht nur beschreibt und erklärt, sondern auch die Landmarken für die Behandlung setzt und zahlreiche neue Perspektiven ermöglicht, fällt er dann eben doch: der Neuschnee. Und was gibt es Schöneres zum Skifahren als eine pulverige Schicht Neuschnee auf einer tragenden Altschneedecke. 


\section{Angaben zum Autor}

Lutz Wittmann, Prof. Dr. phil., Dipl.-Psych., Professor für klinische Psychologie und Psychotherapie an der International Psychoanalytic University Berlin, Stromstrasse 1, 10555 Berlin, Deutschland, lutz.wittmann@ipu-berlin.de 